\title{
Novel features of computer-simulated clonal life of Paramecium caudatum
}

\author{
Tatsuya Uezu ${ }^{1 *}$, Sae Kakutani ${ }^{2}$, Mika Yoshida ${ }^{1}$, \\ Akino NAKAJIMA ${ }^{2}$, Takeshi AsAO ${ }^{1}$, and Yoshiomi TAKAGI ${ }^{3}$
}

Nara Women's University, Nara 630, Japan

${ }^{2}$ Faculty of Sciences, Department of Physics, Nara Women's University, Nara 630, Japan

${ }^{3}$ Nara Women's University, Nara 630, Japan

\section{ABSTRACT}

The clone of the ciliated protozoan Paramecium caudatum has the immaturity period of about 60 fissions and the lifespan of about 600 fissions. These life cycle figures have been depicted through laboratory experiments that allow continuous cell divisions for hundreds, which never occur in nature. We here constructed the nature-mimicking model culture that alternated the log- and stationary phases to allow conjugation, and computer-simulated the age structure modifying parameters such as cell distributions to start the culture, fission rates, death rates, immaturity periods, probabilities of conjugation, proportions of transplantation and so on. The average and maximum ages in the culture after thousands of alternations were converged to $43 \pm 2$ and $140 \pm 5$ fissions, respectively, when parameters for the immaturity period and the maximum clonal lifespan were set at 60 and 600 fissions. This result explains why cells collected in nature are usually young and vigorous. The average and maximum ages proportionally prolonged as the immaturity period was prolonged, as reported true for species of the ciliate. These results indicate the validity of our simulation. The average and maximum ages remained unchanged when the initial condition for starting the culture was changed from two complementary mating-type cells to a population with a quadratic-function

\footnotetext{
*Corresponding author.: +81 74220 3382: fax: +81 742203382

E-mail address: uezu@cc.nara-wu.ac.jp
} 
distribution, and when the fission rate at the log-phase and the death rate at the stationary phase were modified for older ages. The average and maximum ages changed slightly when either the conjugation rate or the proportion of transplantation was somewhat lowered. Although they changed considerably when such parameters as the immaturity period, conjugation rate and death rate were extremely modified, no clones with the age over 230 fissions appeared in any simulations. These results indicate the robustness of the model, which provides us with fresh insight into the structural system of the clonal lifespan of $P$. caudatum in nature.

Keywords:

immaturity period, lifespan, maximum age, average age, model culture

\section{Introduction}

The ciliated protozoa, to which Paramecium belongs, are unique unicellular organisms in that the sexual progeny have the definite division potential or the clonal lifespan similar to that of normal diploid human cells cultured in vitro. The process of asexual reproduction following the sexual reproduction such as conjugation or autogamy is composed of immaturity, maturity and senility: the immaturity period lasts for about 60 fissions, and the clonal lifespan for about 600 fissions in P. caudatum; the autogamy-immaturity period lasts for about 20 fissions, and the clonal lifespan for about 300 fissions in P. tetraurelia. Conjugation or autogamy resets the clonal life to time zero to initiate the new life cycle from immaturity or autogamy-immaturity. The length of each life cycle stage is counted by the

number of fissions, rather than by the physical time (Takagi, 1988). Paramecium that has long been a model organism for cellular aging and clonal lifespan (Sonneborn, 1954, 1974; Smith-Sonneborn, 1981, 1985; Takagi, 1988, 1999) proposes fascinating questions such as whether the maximal lifespan could be extended indefinitely or not, what would happen if the immaturity period was drastically 
shortened or elongated, whether the relationship might exist between the immaturity period and the lifespan, and so forth. These questions have been partly answered by mutant studies. Although the lengths of clonal lifespan (Takagi et al., 1987a) and autogamy-immaturity (Komori et al., 2002) are very variable, the isolation of mutants that have shorter immaturity period in P. caudatum (Myohara \& Hiwatashi, 1978), those that have shorter clonal lifespan in P. tetraurelia (Takagi et al., 1987b), and those that have longer period of autogamy-immaturity in P. tetraurelia (Komori et al., 2004, 2005) indicates that the life cycle stages in Paramecium are genetically regulated. Although it has been reported in mammals (Cutler, 1978) and in ciliates (Smith-Sonneborn, 1981) that the lifespan is directly proportional to the age at sexual maturation, mutants with longer period of autogamy-immaturity were not related to the clonal lifespan (Komori et al., 2004, 2005), suggesting that the interspecies rule between the lifespan and the immaturity period should not be applied to the intraspecies relation (Komori et al., 2005).

Almost all of the findings on the life cycle of Paramecium are based on the laboratory experiments conducted under conditions that never occur in nature. It would be impossible for a Paramecium cell with the size of $10^{-8} \mathrm{~cm}^{3}$ to divide continuously in nature for even 117 times, because the total volume of $2^{117}(\simeq 1.3 \times$ $\left.10^{35}\right)$ cells would exceed the earth volume $\left(1.08 \times 10^{27} \mathrm{~cm}^{3}\right)$. Since the continuous presence of food is necessary for continuous fissions, and since the moderate starvation is a condition to induce the sexual reproduction, the regular transfer of a part of the fission products into new culture medium, usually the daily transfer of one of the fission products called the daily isolation culture, is essential for the life cycle studies in Paramecium. Since the daily isolation culture is possible only in laboratory, the life cycle of Paramecium in nature has remained unrevealed.

To estimate the life features of Paramecium in nature, we here set a model culture of $P$. caudatum that alternates the log-phase and the stationary phase to allow conjugation, and computer-simulated the age structure of the model culture. We first adopted probable numerical values for the parameters such as the cell number at the start of the culture, the fission and death rates at the log-phase, the 
conjugation rate at the stationary phase after a certain length of immaturity period, and the cell numbers at each transfer to a new flask; we then computer-simulated the age structure of the serially transferred populations by modifying these parameters one by one. This is an unprecedented research demonstrating that the length of immaturity and the rate of conjugation in maturity have critical effects on the population profile and why Paramecium cells collected in nature are usually young and vigorous.

\section{Formulation}

\subsection{Model}

The model cells of $P$. caudatum are grown in a flask containing 1,000 ml culture medium that can accommodate $N_{\text {max }}$ cells, starting from an initial population for two complementary mating-type (equivalent to a male and a female) cells, $\mathrm{m}$ and $\mathrm{f}$, at age 0 . The period during which they grow exponentially is referred to as the phase 1, and the period during which they stop dividing, or they are at the stationary phase, is referred to as the phase 2 , in which maturated cells can conjugate. We assume that cells are in the phase 2 for some period $\tau$. Then, $y \%$ of populations are chosen randomly and transplanted into a new flask which contains the same volume of culture medium as in the old flask. We repeat this procedure.

Now, we explain notations. The number of fissions (cell divisions) is represented by $a$, the clonal lifespan by $A^{(\mathrm{s})}(\mathrm{s}=\mathrm{m}$ or $\mathrm{f}$ ), and a pair of $\mathrm{m}$ and $\mathrm{f}$ at their ages $a$ and $a^{\prime}$ by $\left(a, a^{\prime}\right)$. We define the unit time interval $\Delta t=1$ as the period of 1 fission at the phase 1. The number of clones of the age $a$ and the sex s at time $t$ is represented by $N_{t}^{(\mathrm{s})}(a)$, and the total number of clones of the sex s at time $t$ is by $N_{t}^{(\mathrm{s})}=\sum_{a=0}^{A^{(\mathrm{s})}} N_{t}^{(\mathrm{s})}(a)$.

In this paper, we consider the average number of cells. So, we introduce several probabilities in each phase. In the phase 1, probabilities of death, fission, and neither death nor fission for each clone during the time interval $\Delta t=1$ are denoted 
by $p_{\mathrm{d}}^{(\mathrm{s})}(a), p_{\mathrm{b}}^{(\mathrm{s})}(a)$ and $p_{\mathrm{nbd}}^{(\mathrm{s})}(a)$, respectively. These satisfy the following relation,

$$
p_{\mathrm{b}}^{(\mathrm{s})}(a)+p_{\mathrm{d}}^{(\mathrm{s})}(a)+p_{\mathrm{nbd}}^{(\mathrm{s})}(a)=1
$$

During the phase 2 , the probabilities of death and non-death are denoted by $\widetilde{p}_{\mathrm{d}}^{(\mathrm{s})}(a)$ and $p_{\mathrm{nd}}^{(\mathrm{s})}(a)$, respectively. These satisfy the following relation,

$$
\widetilde{p}_{\mathrm{d}}^{(\mathrm{s})}(a)+p_{\mathrm{nd}}^{(\mathrm{s})}(a)=1
$$

These probabilities are supposed to be dependent on age $a$, having no relation to time $t$. The probability of non-death is the sum of the probability of conjugation $p_{\mathrm{c}}^{(\mathrm{s})}(a, t)$ and that of non-conjugation $p_{\mathrm{nc}}^{(\mathrm{s})}(a, t)$,

$$
p_{\mathrm{nd}}^{(\mathrm{s})}(a)=p_{\mathrm{c}}^{(\mathrm{s})}(a, t)+p_{\mathrm{nc}}^{(\mathrm{s})}(a, t)
$$

\subsection{Probabilities of clones that conjugate}

In this subsection, we calculate the probabilities of clones that conjugate.

Suppose that the phase 2 starts at time $t$. Then, the number of clones of the age $a$ and the sex $\mathrm{s}$ that survive at the end of the phase 2 is

$$
M_{t}^{(\mathrm{s})}(a)=p_{\mathrm{nd}}^{(\mathrm{s})}(a) N_{t}^{(\mathrm{s})}(a)
$$

The total number of clones of the sex s at the end of the phase 2 is $M_{t}^{(\mathrm{s})}=$ $\sum_{a=0}^{A^{(\mathrm{s})}} M_{t}^{(\mathrm{s})}(a)$. Then, the maximum number of pairs of male and female at the end of the phase 2 is

$$
M_{\mathrm{p}, t}=\min \left\{M_{t}^{(\mathrm{m})}, M_{t}^{(\mathrm{f})}\right\}
$$

Now, let us assume that the probability at which a pair of clones $\left(a, a^{\prime}\right)$ conjugate during the phase 2 depends on $\left(a, a^{\prime}\right)$. We denote it by $q_{\mathrm{c}}\left(a, a^{\prime}\right)$. When a pair of male and female are chosen in the phase 2 at random, the probability $p_{2}\left(a, a^{\prime}\right)$ that their ages are $\left(a, a^{\prime}\right)$ is estimated as

$$
p_{2}\left(a, a^{\prime}\right)=\frac{M_{t}^{(\mathrm{m})}(a)}{M_{t}^{(\mathrm{m})}} \frac{M_{t}^{(\mathrm{f})}\left(a^{\prime}\right)}{M_{t}^{(\mathrm{f})}}
$$

Therefore, the probability $p_{\mathrm{c}}\left(a, a^{\prime}\right)$ that a pair with ages $\left(a, a^{\prime}\right)$ conjugate is

$$
p_{\mathrm{c}}\left(a, a^{\prime}\right)=q_{\mathrm{c}}\left(a, a^{\prime}\right) p_{2}\left(a, a^{\prime}\right)
$$


Thus, the number of conjugating pairs in which the age of male clones is $a$ is

$$
M_{\mathrm{p}, t} \sum_{a^{\prime}=0}^{A^{(\mathrm{f})}} p_{c}\left(a, a^{\prime}\right)=M_{\mathrm{p}, t} \frac{M_{t}^{(\mathrm{m})}(a)}{M_{t}^{(\mathrm{m})} M_{t}^{(\mathrm{f})}} \sum_{a^{\prime}=0}^{A^{(\mathrm{f})}} M_{t}^{(\mathrm{f})}\left(a^{\prime}\right) q_{\mathrm{c}}\left(a, a^{\prime}\right) .
$$

Expressing this as $p_{\mathrm{c}}^{(\mathrm{m})}(a, t) N_{t}(a)^{(\mathrm{m})}$, we obtain

$$
p_{\mathrm{c}}^{(\mathrm{m})}(a, t)=p_{\mathrm{nd}}^{(\mathrm{m})}(a) \frac{M_{\mathrm{p}, t}}{M_{t}^{(\mathrm{m})} M_{t}^{(\mathrm{f})}} \sum_{a^{\prime}=0}^{A^{(\mathrm{f})}} M_{t}^{(\mathrm{f})}\left(a^{\prime}\right) q_{\mathrm{c}}\left(a, a^{\prime}\right) .
$$

Similarly, we obtain $p_{\mathrm{c}}^{(\mathrm{f})}(a, t)$ as

$$
p_{\mathrm{c}}^{(\mathrm{f})}(a, t)=p_{\mathrm{nd}}^{(\mathrm{f})}(a) \frac{M_{\mathrm{p}, t}}{M_{t}^{(\mathrm{m})} M_{t}^{(\mathrm{f})}} \sum_{a^{\prime}=0}^{A^{(\mathrm{m})}} M_{t}^{(\mathrm{m})}\left(a^{\prime}\right) q_{\mathrm{c}}\left(a^{\prime}, a\right) .
$$

These are the probabilities for clones to conjugate during the phase 2 .

\subsection{Evolution equation for $N_{a, t}^{(\mathrm{s})}$ in the phase 1}

First, let us consider the number of clones of the age $a$ and the sex s at time $t+1$ for $a \geq 1$. During the time interval $\Delta t=1$, a clone of the age $a-1$ and the sex $\mathrm{s}$ at time $t$ duplicates into two clones of the age $a$ and the sex s with the probability $p_{\mathrm{b}}^{(\mathrm{s})}(a-1)$, and a clone of the age $a$ and the sex s survives with the probability $p_{\mathrm{nbd}}^{(\mathrm{s})}(a)$. Thus, the number of clones of the age $a$ and the sex s at time $t+1, N_{a, t+1}^{(\mathrm{s})}$, is given by

$$
N_{t+1}^{(\mathrm{s})}(a)=p_{\mathrm{nbd}}^{(\mathrm{s})}(a, t) N_{t}^{(\mathrm{s})}(a)+2 p_{\mathrm{b}}^{(\mathrm{s})}(a-1) N_{t}^{(\mathrm{s})}(a-1) \quad(a \geq 1) .
$$

Since no clones become age 0 by duplication, the number of clones of the age $a=0$ and the sex $\mathrm{s}$ at time $t+1$ is given by

$$
N_{t+1}^{(\mathrm{s})}(0)=p_{\mathrm{nbd}}^{(\mathrm{s})}(0) N_{t}^{(\mathrm{s})}(0)
$$

\subsection{Evolution equation for $N_{t}^{(\mathrm{s})}(a)$ in the phase 2}

Let us assume that the phase 2 starts at time $t$. The number of the clones of the age $a(\geq 1)$ and the sex s at the end of the phase 2 is that of the clones of the age $a$ and the sex s which survive and do not conjugate during the phase 2, and is given by

$$
N_{t+\tau}^{(\mathrm{s})}(a)=p_{\mathrm{nc}}^{(\mathrm{s})}(a, t) N_{t}^{(\mathrm{s})}(a) \quad(a \geq 1)
$$


The number of the clones of the age $a=0$ is the summation of that of the clones of the age 0 which survive and do not conjugate and that of the clones produced by conjugation during the phase 2 . The former is $p_{\mathrm{nc}}^{(\mathrm{s})}(0, t) N_{t}^{(\mathrm{s})}(0)$. The latter is calculated as follows. The number of conjugating pairs at age $\left(a, a^{\prime}\right)$ is $p_{\mathrm{c}}\left(a, a^{\prime}\right) M_{\mathrm{p}, t}$. Thus, the total number of conjugating pairs $N_{\mathrm{c}, t}$ is

$$
\begin{aligned}
N_{\mathrm{c}, t} & =\sum_{a=0}^{A^{(\mathrm{m})}} \sum_{a^{\prime}=0}^{A^{(\mathrm{f})}} p_{\mathrm{c}}\left(a, a^{\prime}\right) M_{\mathrm{p}, t} \\
& =\frac{M_{\mathrm{p}, t}}{M_{t}^{(\mathrm{m})} M_{t}^{(\mathrm{f})}} \sum_{a=0}^{A^{(\mathrm{m})}} \sum_{a^{\prime}=0}^{A^{(\mathrm{f})}} M_{t}^{(\mathrm{m})}(a) M_{t}^{(\mathrm{f})}\left(a^{\prime}\right) q_{\mathrm{c}}\left(a, a^{\prime}\right) .
\end{aligned}
$$

Thus, we obtain the evolution equation for $N_{t}^{(\mathrm{s})}(0)$ as

$$
\begin{aligned}
N_{t+\tau}^{(\mathrm{s})}(0)= & p_{\mathrm{nc}}^{(\mathrm{s})}(0) N_{t}^{(\mathrm{s})}(0)+N_{\mathrm{c}, t} \\
= & p_{\mathrm{nc}}^{(\mathrm{s})}(0) N_{t}^{(\mathrm{s})}(0) \\
& +\frac{M_{\mathrm{p}, t}}{M_{t}^{(\mathrm{m})} M_{t}^{(\mathrm{f})}} \sum_{a=0}^{A^{(\mathrm{m})}} \sum_{a^{\prime}=0}^{A^{(\mathrm{f})}} M_{t}^{(\mathrm{m})}(a) M_{t}^{(\mathrm{f})}\left(a^{\prime}\right) q_{\mathrm{c}}\left(a, a^{\prime}\right) .
\end{aligned}
$$

\section{Method}

In this section, we describe the method of numerical simulations of evolution equations (11), (12), (13) and (15). Assuming the age dependences of the probabilities $p_{\mathrm{b}}^{(\mathrm{s})}(a), p_{\mathrm{d}}^{(\mathrm{s})}(a)$ in the phase 1 and $\widetilde{p}_{\mathrm{d}}^{(\mathrm{s})}(a), q_{\mathrm{c}}\left(a, a^{\prime}\right)$ in the phase 2 , the probabilities $p_{\mathrm{nbd}}^{(\mathrm{s})}(a), p_{\mathrm{nd}}^{(\mathrm{s})}(a), p_{\mathrm{c}}^{(\mathrm{s})}(a, t)$ and $p_{\mathrm{nc}}^{(\mathrm{s})}(a, t)$ can be calculated by using eqs. (1), (2), (3), (9), (10) and $N_{a, t}^{(\mathrm{s})}$. Thus, giving an initial condition $N_{0}^{(\mathrm{s})}(a)$ for $a=0,1, \cdots, A^{(\mathrm{s})}$, we can obtain the time evolution of $N_{t}^{(\mathrm{s})}(a)$ by (11), (12), (13) and (15). In the numerical simulations, if $N_{t}^{(\mathrm{s})}(a)<1, N_{t}^{(\mathrm{s})}(a)$ is set to 0 .

Here, we explain our choices of system parameters. First of all, we assume that all parameters have the same values for males and females in almost all cases. Thus, hereafter we omit the superscript (s) for all parameters except as otherwise mentioned. The capacity of a flask $N_{\max }$ and the period $\tau$ of the phase 2 are set to $10^{6}$ and 16 , respectively. These are realistic values based on laboratory experiments.

Since we performed simulations by changing initial conditions and several system parameters, we list their values used in this paper. 
A. Initial distribution of population

A-1: delta distribution, consisting of two complementary mating-type cells.

A-2: quadratic distribution for male and female.

A-3: mixed distribution, consisting of the quadratic distribution for male and the delta distribution for female.

B. Immaturity period $a_{\text {imm }}$
B-1: 60 fissions,
B-2: 30 fissions,
B-3: 120 fissions,

B-4: 200 fissions,

B-5: Every age from 30 fissions to 200 fissions.

C. Interval of maximum conjugation probability $a_{\text {int }}$

C-1: 140 fissions, $\quad$ C-2: 100 fissions, $\quad$ C-3: 40 fissions.

D. Maximum of conjugation probability $c(a), c_{\max }$
D-1: 0.7 ,
D-2: 0.4
D-3: 0.1 .

E. Ratio $y$ of transplantation from the phase 2 to the phase 1
E-1: $y=1[\%]$,
E-2: $y=0.1[\%]$.

F. Fission probability, $p_{\mathrm{b}}(a)$

F-1 : linear decrease from 0.9 at age 0 to 0.6 at age 400 , and then to 0 at age 600 fissions.

F-2 : ditto except during the period from age 400 to 600 fission, in which 0.6 is kept unchanged.

F-3 : smaller $p_{\mathrm{b}}(a)$ than that in F-1; linear decrease from 0.6 at age 0 to 0.3 at age 400, then to 0 at age 600 fissions.

G. Death probability, $p_{\mathrm{d}}(a), \widetilde{p}_{\mathrm{d}}(a)$

G-1 : $p_{\mathrm{d}}(a) ; 0.01$ until age 200 fissions, then linear increase to 0.2 at age 400 and then to 1 at age 600 fissions, $\widetilde{p}_{\mathrm{d}}(a) ; 0.01$ until age 200 fissions, then linear increase to 0.3 at age 400 and then to 1 at age 600 fissions. 
G-2 : $p_{\mathrm{d}}(a)=\widetilde{p}_{\mathrm{d}}(a) ; 0.01$ throughout the lifetime.

G-3 : larger $p_{\mathrm{d}}(a)$ and $\widetilde{p}_{\mathrm{d}}(a)$ than those in G-1.

$p_{\mathrm{d}}(a) ; 0.09$ until age 200 fissions, then linear increase to 0.3 at age 400 and then to 1 at age 600 fissions,

$\widetilde{p}_{\mathrm{d}}(a) ; 0.2$ until age 200 fissions, then linear increase to 1 at age 600 fissions.

G-4 : larger $p_{\mathrm{d}}(a)$ and $\widetilde{p}_{\mathrm{d}}(a)$ than those in G-3.

$p_{\mathrm{d}}(a)$; linear decrease from 0.3 at age 0 to 0.2 at age 200 , and 0.2 until age 400, then linear increase to 1 at age 600 fissions,

$\widetilde{p}_{\mathrm{d}}(a)$; linear decrease from 0.7 at age 0 to 0.5 at age 200 , and 0.5 until age 400, then linear increase to 1 at age 600 fissions.

The default parameter set is A-1, B-1, C-1, D-1, E-1, F-1 and G-1. In the below, we explain the default parameters in detail.

\section{Initial distribution, A-1: Delta distribution}

As an initial distribution, we take a pair of male and female.

$$
\begin{aligned}
N_{0}^{(\mathrm{m})}(a) & =\delta_{0, a}, \\
N_{0}^{(\mathrm{f})}(a) & =N_{0}^{(\mathrm{m})}(a),
\end{aligned}
$$

where $\delta_{0, a}$ is the Kronecker's delta, which is 1 for $a=0$ and 0 otherwise. We call this the delta distribution.

\section{Parameters concerning conjugation probability, B-1, C-1, D-1}

It is plausible to consider that the probability that a pair of clones $\left(a, a^{\prime}\right)$ meet is mainly determined by the more mobile clone. Thus, we assume that $q_{\mathrm{c}}\left(a, a^{\prime}\right)$ depends only on the age of the younger clone. Further, we assume that a male and a female can conjugate only when their ages are in a definite range, $a_{\text {imm }}<a<a_{\text {old }}$. That is, the period $0 \leq a \leq a_{\text {imm }}$ is the immature period during which clones can not conjugate, and the period $a_{\text {old }} \leq a \leq A$ is the old period during which clones are considered too senile to conjugate. Concretely, $q_{\mathrm{c}}\left(a, a^{\prime}\right)$ is assumed as follows.

$$
q_{c}\left(a, a^{\prime}\right)= \begin{cases}c(a) & \left(a \leq a^{\prime}\right) \\ c\left(a^{\prime}\right) & \left(a>a^{\prime}\right)\end{cases}
$$


We adopt a piecewise linear function for $c(a)$ as follows.

$$
c(a)= \begin{cases}0 & \left(0 \leq a \leq a_{\mathrm{imm}}\right) \\ c_{\max } & \left(a_{\mathrm{imm}}<a \leq a_{\mathrm{imm}}+a_{\mathrm{int}}\right) \\ c_{\max }+\frac{c_{\max }}{200}\left(a_{\mathrm{imm}}+a_{\mathrm{int}}-a\right) & \left(a_{\mathrm{imm}}+a_{\mathrm{int}}<a \leq a_{\mathrm{imm}}+a_{\mathrm{int}}+200\right) \\ 0 & \left(a_{\mathrm{imm}}+a_{\mathrm{int}}+200<a \leq 600\right) .\end{cases}
$$

Here, $a_{\mathrm{int}}$ is the interval during which $c(a)$ is maximum, and $a_{\mathrm{old}}=a_{\mathrm{imm}}+a_{\mathrm{int}}+200$.

We set the default values as $a_{\mathrm{imm}}=60, a_{\mathrm{int}}=140$ and $c_{\max }=0.7$. See Fig. 1 .

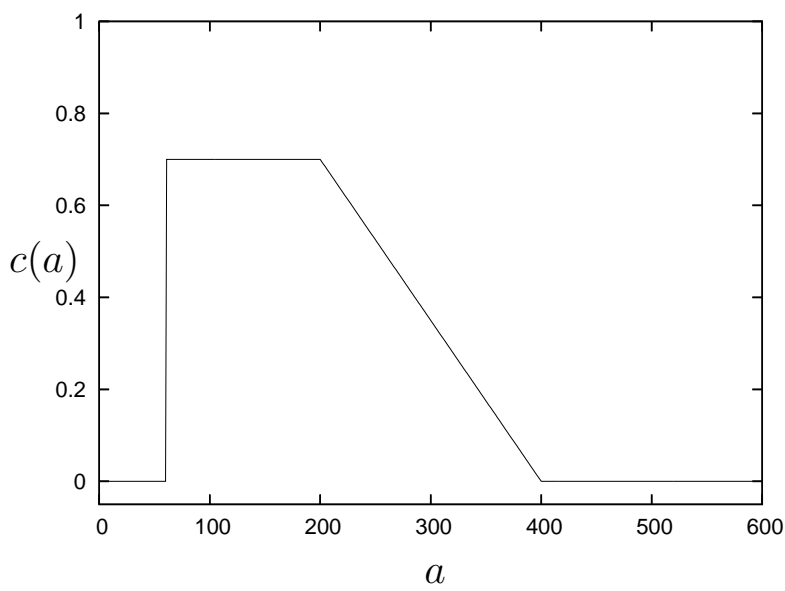

Figure 1: Age dependence of $c(a)$.

Ratio $y$ of transplantation from the phase 2 to the phase 1, E-1

$y=1[\%]$ is plausible value for laboratory experiments.

\section{Fission and death probabilities, F-1, G-1}

Here, we assume that $p_{\mathrm{b}}(a)$ is a non-decreasing function and $p_{\mathrm{d}}(a)$ and $\widetilde{p}_{\mathrm{d}}(a)$ are non-increasing functions with respect to $a$. For simplicity, we adopt the piecewise linear functions.

\section{Results}

Table 1 shows the simulation results, i.e., the converged values, for the average age $a_{\mathrm{av}}$ and the maximum age $a_{\mathrm{max}}$.

Table 1. Simulation results for the average age $a_{\text {av }}$ and the maximum age $a_{\max }$. The top column shows the results for the default parameter set, and the others show the results when one of the parameters is changed from the default. 


\begin{tabular}{lcc}
\hline Parameters & $a_{\mathrm{av}}$ & $a_{\mathrm{max}}$ \\
\hline Default, A-1, B-1, C-1, D-1, E-1, F-1, G-1 & $43 \pm 2$ & $140 \pm 5$ \\
\hline A-2: Delta distribution $\rightarrow$ Quadratic distribution & $43 \pm 2$ & $140 \pm 5$ \\
\hline B-2: $a_{\text {imm }}=60 \rightarrow a_{\text {imm }}=30$ & $26 \pm 3$ & $106 \pm 5$ \\
\hline B-3: $a_{\text {imm }}=60 \rightarrow a_{\text {imm }}=120$ & $80 \pm 30$ & $165 \pm 45$ \\
\hline B-4: $a_{\text {imm }}=60 \rightarrow a_{\text {imm }}=200$ & $110 \pm 50$ & $170 \pm 50$ \\
\hline C-2: $a_{\text {int }}=140 \rightarrow a_{\text {int }}=100$ & $43 \pm 2$ & $140 \pm 5$ \\
\hline C-3: $a_{\text {int }}=140 \rightarrow a_{\text {int }}=40$ & $43 \pm 2$ & $140 \pm 5$ \\
\hline D-2: $c_{\max }=0.7 \rightarrow c_{\max }=0.4$ & $48 \pm 2$ & $152 \pm 5$ \\
\hline D-3: $c_{\max }=0.7 \rightarrow c_{\max }=0.1$ & $65 \pm 2$ & $188 \pm 5$ \\
\hline E-2: $y=1 \% \rightarrow y=0.1 \%$ & $45 \pm 5$ & $117 \pm 8$ \\
\hline F-2: $p_{\mathrm{b}}=0.6$ to 0 for $a \in(400,600) \rightarrow p_{\mathrm{b}}=0.6$ for $a \in(400,600)$ & $43 \pm 2$ & $140 \pm 5$ \\
\hline G-2: $p_{\mathrm{d}}, \widetilde{p}_{\mathrm{d}}=0.01$ for $a \in(0,200) \rightarrow p_{\mathrm{d}}, \widetilde{p}_{\mathrm{d}}=0.01$ for $a \in(0,600)$ & $43 \pm 2$ & $140 \pm 5$ \\
\hline G-3: larger $p_{\mathrm{d}}$ and $\widetilde{p}_{\mathrm{d}}$ than those of G-1 & $46 \pm 4$ & $144 \pm 5$ \\
\hline F-3 and G-4: smaller $p_{\mathrm{b}}$ than that of F-1 & $52 \pm 6$ & $167 \pm 13$ \\
and larger $p_{\mathrm{d}}$ and $\widetilde{p}_{\mathrm{d}}$ than those of G-3 & \\
\hline
\end{tabular}

In the following subsections (4.1-4.5), we describe the simulation results for $a_{\mathrm{av}}$ and $a_{\max }$ when one of the parameters is changed from the default value except as otherwise mentioned.

\subsection{Initial condition dependence}

First, we compare the delta distribution A-1 with the following quadratic distribution A-2 for males and females,

$$
\begin{aligned}
N_{0}^{(\mathrm{m})}(a) & =200 \frac{a}{300}\left(\frac{600-a}{300}\right), \\
N_{0}^{(\mathrm{f})}(a) & =N_{0}^{(\mathrm{m})}(a) .
\end{aligned}
$$

Since all parameters and initial distributions of population are same for males and females, populations of male and female cells are equal at any time.

Fig. 2 shows how $a_{\text {av }}$ and $a_{\max }$ are converged irrespective of initial conditions. For the delta distribution, A-1, $a_{\max }$ initially increases as time passes and reaches the max value 160 at $t=490$, when $a_{\text {av }}$ is 37.6. Just after this, the transplantation takes place and at $t=506, a_{\max }$ becomes 102 , when $a_{\mathrm{av}}$ is 35.7 . The reason for these is considered as follows. After conjugation, only $1 \%$ of populations are transplanted and if $N_{t}(a)$ is less than 1 , it is set to 0 . By this procedure, clones of higher ages 


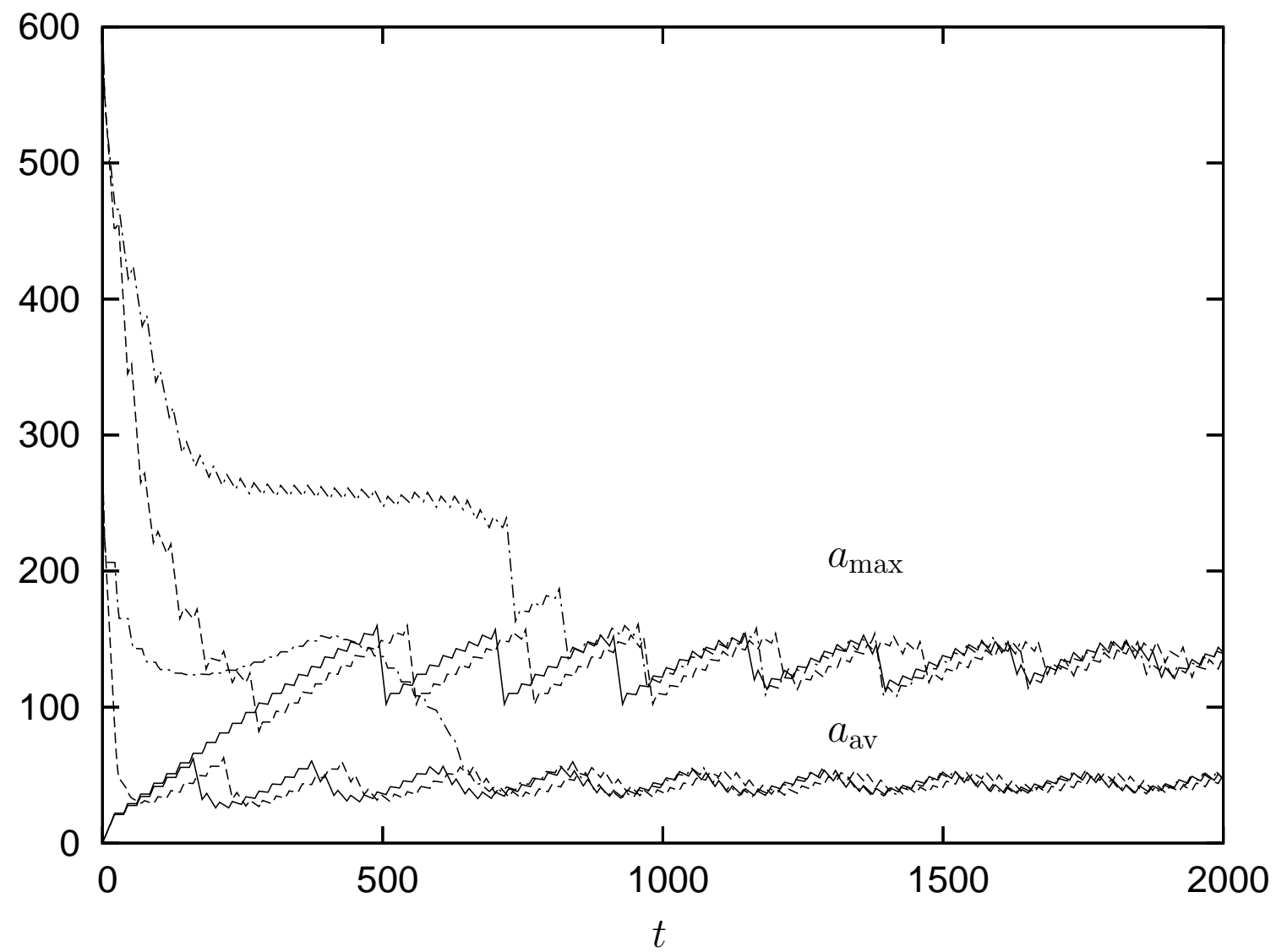

Figure 2: Time series of average age $a_{\mathrm{av}}$ and maximum age $a_{\max }$ for males.

Solid curve: delta distribution (A-1), dashed curve: quadratic distribution (A-2), dashed-dotted curve: mixed distribution (A-3).

are removed and the maximum age becomes lower. Since the number of removed population is small, the average age changes only a little. We note that as time passes, the fluctuations of $a_{\max }$ and $a_{\mathrm{av}}$, denoted by $\Delta a_{\max }$ and $\Delta a_{\mathrm{av}}$ respectively, become smaller and finally become constant values of approximately 5 and 2 . On the other hand, behaviors of $a_{\max }$ and $a_{\mathrm{av}}$ for the quadratic distributions, A-2, are different from those for the delta distribution until about $t=600$. Later, however, $a_{\max }$ and $a_{\text {av }}$ behave similarly to those for the delta distribution.

These results are obtained under the condition that initial distributions for males and females are the same. In order to study the effect of different initial distributions between males and females, we performed numerical simulations with the quadratic distribution (eq.(19)) for males, and the following delta distribution for females,

$$
N_{0}^{(\mathrm{f})}(a)=n_{0} \delta_{0, a} .
$$

We call this the mixed distribution, A-3. We studied the system behaviors for A-3 and found that clones die in a finite time for $n_{0} \leq 5$, whereas similar behaviors as 
before are observed for $n_{0}>5$. We performed numerical simulations for $n_{0}=10$ and found that both $a_{\max }$ and $a_{\text {av }}$ for males and females coincide until $t=1000$. In Fig. 2, $a_{\max }$ and $a_{\mathrm{av}}$ for males are displayed for A-3 together with for A-1 and A-2. As time passes, behaviors of $a_{\max }$ and $a_{\mathrm{av}}$ become similar for these three cases, although their initial behaviors are different.

One of the remarkable features of the system is that the values of the average and maximum ages are very small: $a_{\mathrm{av}}=43 \pm 2$ and $a_{\max }=140 \pm 5$, respectively. This implies that most cells are immature, and the maximum age is far below the clonal lifespan $A=600$ revealed in the laboratory.

Next, in order to study what determines $a_{\mathrm{av}}$ and $a_{\mathrm{max}}$, we performed numerical simulations by changing the system parameters, B-G.

\section{2 $a_{\mathrm{imm}}$ and $a_{\mathrm{int}}$ dependence}

We studied the dependence of $a_{\max }$ and $a_{\mathrm{av}}$ on the immature period $a_{\mathrm{imm}}$ and the interval $a_{\text {int }}$ during which the conjugation probability $c(a)$ remains to be maximum. $a_{\mathrm{imm}}$ is set to 30,60 and 120 , and $a_{\text {int }}$ is set to 40,100 and 140 . Numerical simulations were performed for all combinations of $a_{\mathrm{imm}}$ and $a_{\mathrm{int}}$ with other parameters fixed to the default values. We found that the behavior of the system depends on $a_{\text {imm }}$ but not on $a_{\text {int }}$.
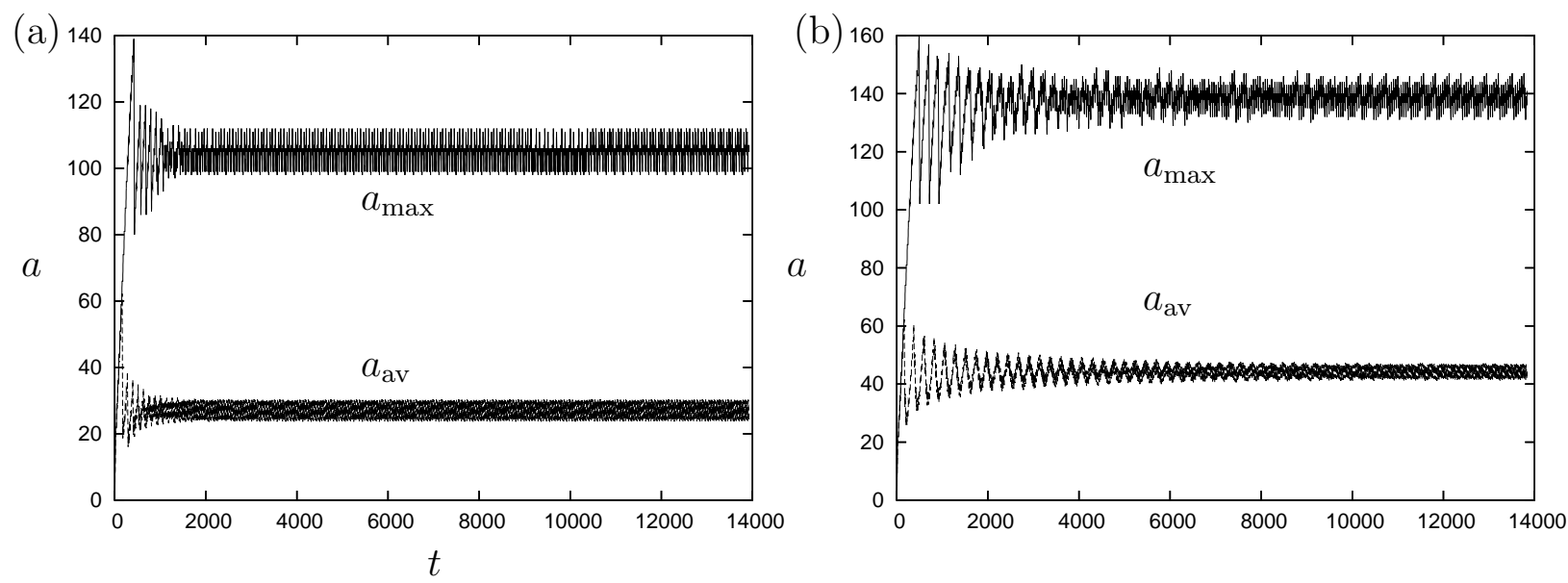

In Fig. 3, we show the time series of $a_{\max }$ and $a_{\mathrm{av}}$ for three values of $a_{\mathrm{imm}}$. We note that the larger $a_{\mathrm{imm}}$ is, the larger both $a_{\mathrm{av}}$ and $a_{\mathrm{max}}$ are. Further, fluctuations for $a_{\mathrm{av}}$ and $a_{\max }$ are similar and small for $a_{\mathrm{imm}}=30$ and 60 , whereas they become 


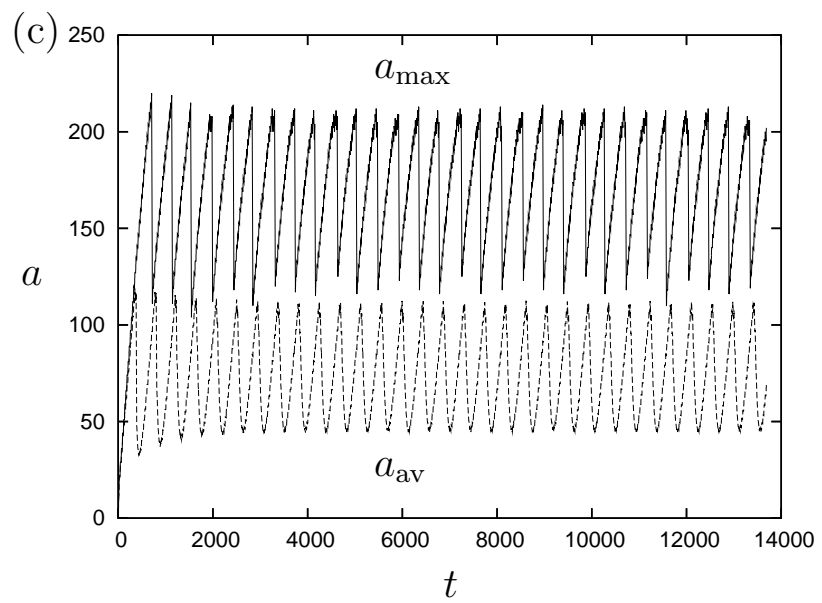

Figure 3: Time series of the maximum age $a_{\max }$ and the average age $a_{\mathrm{av}}$. Solid curve: $a_{\max }$, dashed curve: $a_{\mathrm{av}} \cdot a_{\text {int }}$ is fixed to 140 .

(a) $a_{\mathrm{imm}}=30$, (b) $a_{\mathrm{imm}}=60$, (c) $a_{\mathrm{imm}}=120$.

quite large for $a_{\mathrm{imm}}=120$. Since ages of clones become 0 when conjugation takes place, the former result is explicit because of the frequent occurrence of conjugation. The reason for the latter result is considered as follows. For $a_{\text {imm }}=120$, a gap is observed in $N_{t}(a)$ at the beginning of the phase 1 . As time passes, the older generation group disappears at some time, then the maximum age discontinuously decreases. This causes large fluctuations in $a_{\mathrm{av}}$ and $a_{\mathrm{max}}$. On the other hand, no such a gap is observed for $a_{\text {imm }}=30$ and 60 . Thus, the period of the oscillation of $a_{\mathrm{av}}$ and $a_{\max }$ for $a_{\mathrm{imm}}=30$ and 60 is different from that for $a_{\mathrm{imm}}=120$. The former is approximately 23 which is the period of the transplantation but the latter is approximately 43 which is the time interval for the cells with the age $a_{\max }-\Delta a_{\max }$ to become the age $a_{\max }+\Delta a_{\max } \cdot \Delta a_{\max }$ is not independent of $a_{\mathrm{imm}}$ but the period of the oscillation and $\Delta a_{\max }$ are determined simultaneously.

In order to study the immature period dependences of $a_{\mathrm{av}}$ and $a_{\max }$ in more detail, we calculated them by increasing $a_{\text {imm }}$ from 30 to 200 with the increment 1. As shown in Fig. $4, a_{\mathrm{av}}$ is approximately proportional to $a_{\mathrm{imm}}$, whereas $a_{\mathrm{max}}$ saturates and becomes approximately 170 at about $a_{\mathrm{imm}}=110$. The standard deviations for $a_{\mathrm{av}}$ and $a_{\max }$ become gradually larger as $a_{\text {imm }}$ increases.

The reason why $a_{\max }$ saturates is related to the behaviors of death probabilities $p_{d}(a)$ and $\widetilde{p}_{d}(a)$. They are set constant until $a=200$ but increasing for $a>200(\equiv$ 


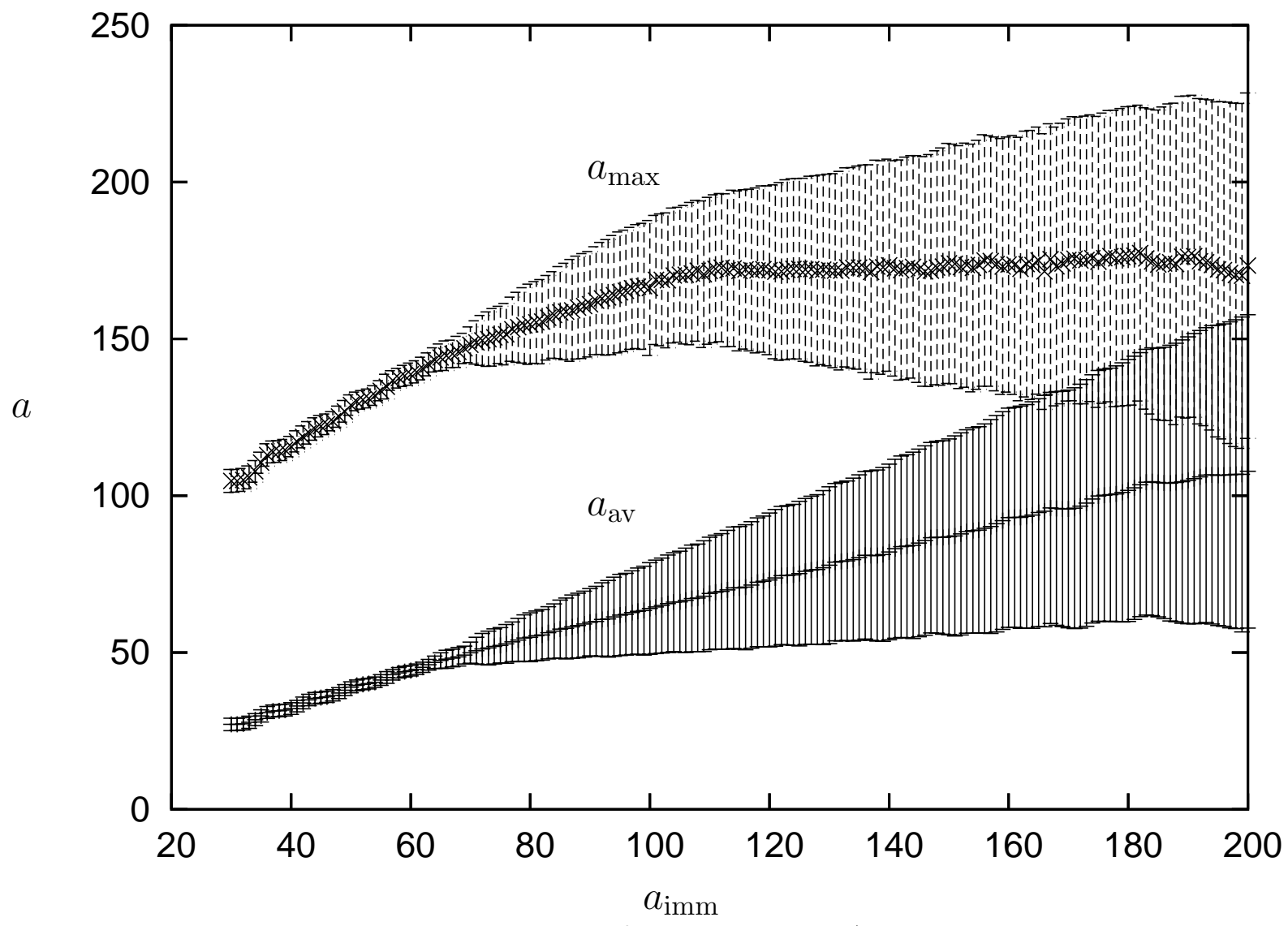

Figure 4: Immature period dependence of $a_{\mathrm{av}}$ and $a_{\mathrm{max}}$. Average over

3,000 iterations which correspond to 2,000 fissions, and the initial 2,000 iterations are not included in the simulations of the average and standard deviation. Vertical lines denote standard deviations. Solid curve: $a_{\mathrm{av}}$, dashed curve: $a_{\max } \cdot a_{\text {int }}$ is fixed to 140 .

$\left.a_{d}\right)$. Thus, If $a_{\max }+\Delta a_{\max }$ exceeds $a_{d}$, the number of oldest cells decreases more rapidly and by the transplantation procedure, the number of these cells is set to 0 if it is less than 1 . Let $a_{\mathrm{imm}, \mathrm{s}}$ be the immature period where $a_{\max }$ saturates. It is estimated from the following relation,

$$
a_{\max }+\Delta a_{\max }=a_{d}
$$

From Fig. 4, we obtain $a_{\text {imm, s }} \simeq 120$, which is consistent with the value 110 estimated above.

\subsection{Maximum conjugation probability dependence}

We found that $a_{\mathrm{av}}$ and $a_{\max }$ become larger as $c_{\max }$ is smaller (Table 1: D-2 and D-3). This is reasonable because if conjugation takes place less frequently, mature cells which do not conjugate increase so that the age 0 cells decrease. 


\subsection{Transplantation ratio dependence}

We found that the smaller the transplantation ratio $(y)$ is, the longer the average age and the shorter the maximum age are, respectively. (Table 1: E-2). The reasons for these are considered as follows. If $y$ is smaller, older cells are removed more, because we set $N_{t}(a)=0$ if $N_{t}(a)<1$. This causes smaller $a_{\max }$ by the reduction of $y$. On the other hand, $N_{t}(a)$ oscillates as the function of $a$ with $t$ fixed. In particular, for $a$ smaller than $a_{\mathrm{av}}$, there are ages of $N_{t}(a)=0$. Thus, cells of younger ages also removed more. Therefore, there are two factors by the reduction of $y$, one is increasing $a_{\mathrm{av}}$, and the other is decreasing $a_{\mathrm{av}}$. As a whole for the present parameter, $a_{\text {av }}$ increases only a little.

\subsection{Fission and death probabilities dependence}

In F-2, $p_{\mathrm{b}}(a)$ is kept 0.6 for $a \in(400,600)$ and in $\mathrm{G}-2, p_{\mathrm{d}}(a)$ and $\widetilde{p}_{\mathrm{d}}(a)$ are kept 0.01 throughout the lifetime. We found that $a_{\mathrm{av}}$ and $a_{\max }$ for F-2 or for G-2 with other parameters fixed to the default values are the same as those for the default parameter set. This is because the probabilities during the interval $(200 \sim 600)$ are not relevant since $a_{\max }$ is at most 150 .

Next, we consider very severe circumstances by assuming high death probabilities that might occur in some natural circumstances. We increased death probabilities $p_{\mathrm{d}}(a)$ and $\widetilde{p}_{\mathrm{d}}(a)$ for $a \leq 2009$ times and 20 times larger respectively than each of the default values. This is G-3. The simulation result shows that both $a_{\mathrm{av}}$ and $a_{\max }$ are slightly larger values than those for the default parameters. Finally, we change not only death probabilities but also the fission probability. The fission probability is decreased from the default value. This is F-3. We assume that death probabilities $p_{\mathrm{d}}(a)$ and $\widetilde{p}_{\mathrm{d}}(a)$ are monotonically decreasing functions for $a \leq 200$ with larger values than those in G-3. This is G-4. We performed simulations with doubly modified parameters of F-3 and G-4, in which the circumstance is severer than in G-3. The result shows a considerably larger $a_{\text {av }}$ and $a_{\max }$ than those for G-3. The reason for these are considered as follows. The larger the death probability in the phase 1 is, 
the more time it takes for the number of cells to saturate. As a result, the period of the phase 1 becomes longer and it takes more time for cells to conjugate, that is, cells become older when the phase 2 starts. Further, the increase of the period of the phase 1 causes the longer interval for the successive conjugation.

From the simulation results shown above, we conclude that $a_{\mathrm{av}}$ and $a_{\mathrm{max}}$ depend strongly on the immature period $a_{\mathrm{imm}}$ and the maximum conjugation probability $c_{\max }$, slightly on the transplantation rate $y$. On the other hand, they do not change drastically when death probabilities are reduced considerably. They remain unchanged in A-2, C-2, C-3, F-2 and G-2 (Table 1).

\section{Discussion}

We here challenged for the first time to estimate the life features of Paramecium in nature. We constructed a population dynamics model of P. caudatum taking into account conjugation. We first set the default parameters as follows: two paramecia cells of one male and one female were allowed to start their life cycle in a flask containing 1,000 ml culture medium; they divided exponentially at the rate of 0.9 with the death rate of 0.01 (phase 1) until reaching $10^{6}$ cells, and starved for 4 days $(\tau=16)$ with the death rate of 0.01 and conjugated at the rate of 0.7 if both sexes were mature or over 60 fissions old (phase 2). One $\%$ of the phase 2 population was transplanted to a new flask containing 1,000 $\mathrm{ml}$ culture medium to restart the new life cycle from the phase 1 . They were defined to advance 1 age at 1 fission, and to rejuvenate to age 0 by conjugation.

We derived evolution equations for the population at time $t$ and age $a$ and sex s, $N_{t}^{(\mathrm{s})}(a)$, and performed numerical simulations of the evolution equations by using the default and optional parameters. The number of cells which conjugate was calculated under several assumptions. We calculated the age distribution, average and maximum ages $\left(a_{\mathrm{av}}, a_{\max }\right.$, respectively) in a population at time $t$.

We found that the average and maximum ages were converged to $43 \pm 2$ and $140 \pm 5$ fissions under conditions with the default parameters. These numbers show that average cells are immature and the most elderly cells are still vigorous, because 
the immaturity period and the maximum clonal lifespan were set at 60 and 600 fissions, respectively. This situation is consistent with our experiences: when the laboratory stocks become old and weaken we go collect samples from nature, which are usually young and vigorous. We can say, therefore, that our model with the default parameters is considered reasonable in mimicking the nature.

What was novel in this study was that we were able to examine for the first time which parameters would affect most the age structure and to what extent each parameter was effective. Among all of the parameters examined, the length of immaturity period or the age at sexual maturation was pivotal to change the age structure. Other parameters such as the number of cells constituting the starting culture, the proportion of the transplantation, the fission probability and the death probability at the phase 1, except for the probability of conjugation, contributed little, if anything. By changing the probability of conjugation from 0.7 to 0.1 , the average and maximum ages were raised and converged to $65 \pm 2$ and $188 \pm 5$ fissions.

The parameters such as the death rate and the conjugation rate would change drastically in nature depending on the presence or absence of the predators and the mating partners. The default value of 0.01 for the death rate could be too low in nature because paramecia would fall prey of many predators. We then tried simulation by increasing the death rate 9 times larger to 0.09 in the phase 1 and 20 times larger to 0.2 in the phase 2 (G-3): the average and maximum ages were converged to $46 \pm 4$ and $144 \pm 5$ fissions. Even the death rate far more severer than this (F-3 and G-4) resulted in the convergence of the average and maximum ages barely to $52 \pm 6$ and $167 \pm 13$ fissions. This indicates that the death rate is not the parameter of much importance for the age structure.

The parameter values we used in this paper are set as real as possible especially for the default parameters. The immaturity period $a_{i m m}=60$ and the clonal lifespan $A^{(\mathrm{s})}=600$ are the observed values for $P$. caudatum. Other default parameters such as $a_{\text {int }}, c_{\max }, p_{\mathrm{b}}(a), p_{\mathrm{d}}(a)$ and $\widetilde{p}_{\mathrm{d}}(a)$ also appear plausible from our experimental studies, although the optional values are arbitrary. In order to investigate whether the system is structurally stable or not, we performed simulations changing these 
parameters over a wide range. As a result, we found that the system behaviors are quite robust by the change of these parameters.

Science of the lifespan faces to some difficult problems. For example, the lifespan observed in nature are not identical to that observed under human control, as exemplified by the big difference of the lifespan between a wild animal and the same animal in a zoo or an aquarium. Besides, the lifespan of some organisms such as Paramecium can be studied only in laboratory, where experiments are conducted in a condition that never occurs in nature so that few data are available for their natural features. These problems have been overcome by this computer simulation study. However, one problem remains to be answered: what kind of world does our simulation results represent, interspecies world or intraspecies world? The interspecies rule should be distinguished from the intraspecies rule. For example, the equation of $T_{\text {met }}=k W^{0.25}$, where $T_{\text {met }}$ is the physiological time including the lifespan and $W$ is the body mass, can be applied to mammalian species (Schmidt-Nielsen, 1984), but organisms with bigger $W$ do not assure the longer lifespan among individuals of the same species as seen in humans. Our simulations using different values for the immaturity period should represent the interspecies relation in nature, because the immaturity period and lifespan changed proportionally as suggested from the interspecies relation (Smith-Sonneborn, 1981), contrasting with the independency of the two factors revealed by our intraspecies mutant study (Komori et al., 2005).

Simulations of our model were not only realistic but also robust and innovative as shown by a stable behavior under different parameters and an emergence of the unexpected age structure. We are thus provided for the first time with fresh insight into the life of $P$. caudatum in nature.

\section{References}

Cutler, R. G., 1978. Evolutionary biology of senescence. In: Behnke, J. A., Finch, C. E., Moment, G. B. (Eds.) The Biology of Aging. Plenum Press, New York and 
London. 311-360.

Komori, R., Harumoto, T., Fujisawa, H., Takagi, Y., 2002. Variability of autogamy-maturation pattern in genetically identical populations of Paramecium tetraurelia. Zool. Sci. 19, 1245-1249.

Komori, R., Harumoto, T., Fujisawa, H., Takagi, Y., 2004.

A Paramecium tetraurelia mutant that has long autogamy immaturity period and short clonal life span. Mech. Ageing Dev. 125, 603-613.

Komori, R., Sato, H., Harumoto, T., Takagi, Y., 2005. A new mutation in the timing of autogamy in Paramecium tetraurelia. Mech. Ageing Dev. 126, 752-759.

Myohara, K., Hiwatashi, K., 1978. Mutants of sexual maturity in Paramecium caudatum, selected by erythromycin resistance. Genetics 90, 227-241.

Schmidt-Nielsen, K. 1984. Scaling. Cambridge University Press, Cambridge.

Smith-Sonneborn, J., 1981. Genetics and aging in protozoa. Int. Rev. Cytol. 73, 319-354.

Smith-Sonneborn, J., 1985. Aging in unicellular organisms. In: Finch, C. E., Schneider, C.L. (eds.) Handbook of the Biology of Aging, Second Edition. Van Nostrand Reinhold, New York, pp. 79-104.

Sonneborn, T. M., 1954. The relation of autogamy to senescence and rejuvenescence in Paramecium aurelia. J. Protozool. 1, 38-53.

Sonneborn, T. M., 1974. Paramecium aurelia. In: Mayr, E., (ed.) Handbook of Genetics, Vol.2. Plenum, New York, London, pp. 433-467. 
Takagi, Y., 1988. Aging. In: Görtz, H-D., (ed.) Paramecium. Springer-Verlag, Berlin, pp. 131-140.

Takagi, Y., 1999. Clonal life cycle of Paramecium in the context of evolutionally acquired mortality. In: Macieira-Coelho, A., (ed.) Cell Immortalization. Springer-Verlag, Berlin, pp. 81-101.

Takagi, Y., Nobuoka, T., and Doi, M. 1987a. Clonal lifespan of Paramecium tetraurelia: effect of selection on its extension and use of fissions for its determination. J. Cell Sci. 88, 129-138.

Takagi, Y., Suzuki, T., Shimada, C., 1987b. Isolation of a Paramecium tetraurelia mutant with short clonal life-span and novel life-cycle features. Zool. Sci. 4, 73-80. 